\title{
Design and Implementation of Internship Evaluation System Based on ASP.NET
}

\author{
Ying Zhang \\ Department of Teaching Affairs Jilin Agricultural University, 130118, Changchun, China \\ jlauzy@126.com
}

Keywords: ASP.NET; tri-level B/S structure; internship evaluation system

\begin{abstract}
In this paper, we designed an internship evaluation system by ASP.NET technology, conducted a detailed analysis and design of the proposed development system function, the establishment of ER model diagram, database structure, analysis of the B / S three-tier architecture model framework to build a model of the framework internship evaluation system, focusing on analysis of the functional module ASP implementation process, the success achieve ASP runtime environment based the Web internship evaluation system development and design.
\end{abstract}

\section{Introduction}

As a college and university, practical ability of students is our school students is an important direction, is currently done in a weak link. As the specialty practice teaching, use of traditional assessment tests are hard to quantify, so we developed the internship evaluation system. This paper aims to use ASP.NTE technique and SQL Server database to develop a convenient, efficient and rationally-designed internship evaluation system. The internship evaluation system is mainly targeted at the institutions of higher learning, making teachers realize their strengths and weaknesses in teaching and immediately improve their teaching methods and strategies so as to achieve better teaching effect.

\section{The introduction of ASP.NTE technique}

ASP.NTE is a widely-used Server script technique. It can be embedded into the script of the webpage and implemented by the Server. The document edited with ASP.NTE technique is suffixed with “aspx.” When the user opens an aspx document, IIS will analyze and implement the document step by step on the Server. Finally, the aspx document will return to the browser in the pure HTML form. ASP.NET technique boasts the characteristics of efficiency and good safety[1].

\section{The design and realization of the internship evaluation system}

The system adopts a three-layer model based on B/S system structure. ASP.NET technique serves as its development environment. Based on SQL Server database, it achieves the adding, checking and other functions of the internship evaluation record.

The system is mainly divided into three layers, namely standing for the presentation layer, business logic layer and data access layer. To put it specifically[2],

- Presentation layer: It is mainly to provide an interaction graph interface for users. It receives the data submitted by the user and present the processed data sent by the Server to users.

- Business logic layer: It is mainly made up of Web form and XML Web service, which is a bridge for the data exchange between the data access layer and the presentation layer.

- Data access layer: It is mainly to add, delete, modify, store and retrieve data and return the retrieved result.

The three-layer structure chart of the system is shown in Figure 1. 


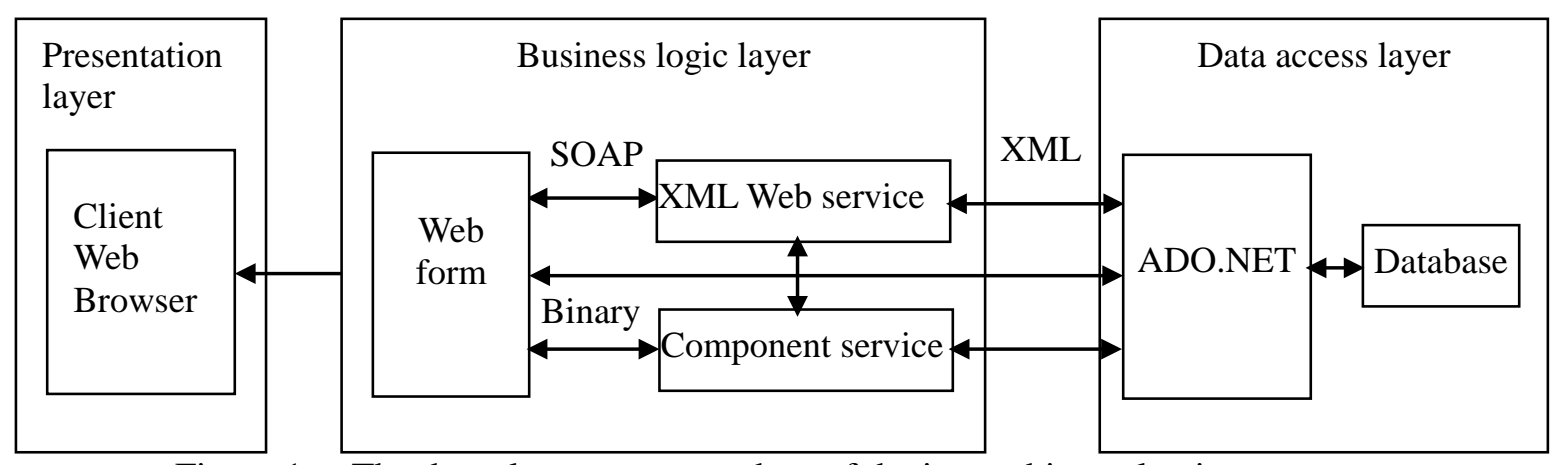

Figure 1. The three-layer structure chart of the internship evaluation system

\section{Design of system's functional modules}

Major function modules of the system are shown in Figure 2.

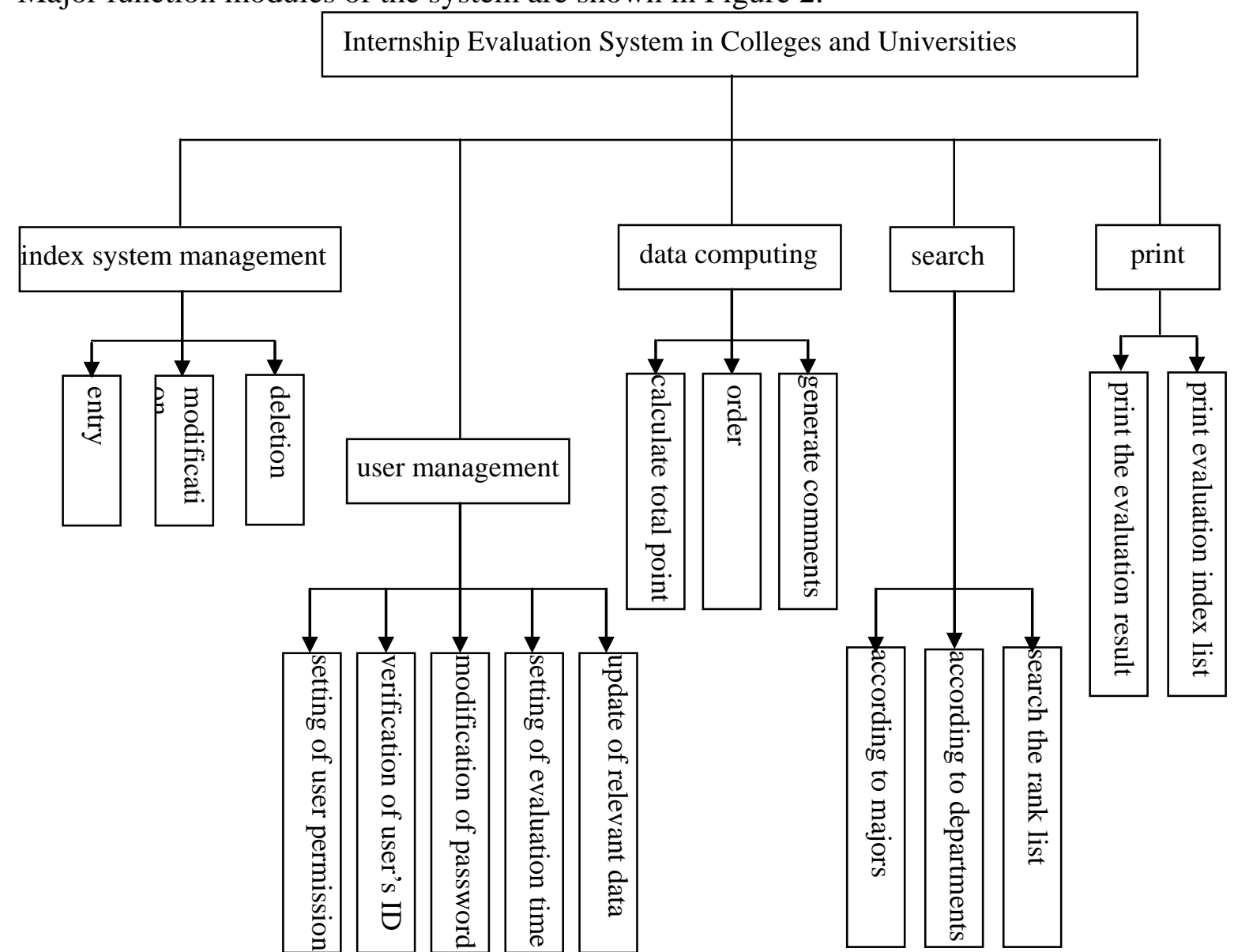

Figure 2. Major function modules of internship evaluation system

System is mainly for teachers, students, courses conducted three comprehensive examination, for each evaluation subjects, the system design multiple indicators. Administrators can modify the contents and weight of each indicator in the background. Through systematic evaluation, teachers can better understand the actual students and found that the process of teaching the issues in order to better on its own way tutorial method for reflection and improvement; student's final grade is also the decision not just by test scores, also depends on the students in the course of learning attitude and practice capability, students can better promote the overall quality of a comprehensive upgrade their level; for the evaluation of courses improvement courses for college structure provides a reference.

In the system design process, taking into account the users of the system include: administrators, teachers, students, experts, and their computer operations in various countries, so try to let the system be intuitive interface, easy operation, stable, safe and reliable, set up an interactive platform to achieve the unity of summative assessment and evaluation form[3]. 
The internship evaluation system is designed and achieved through the following functions:

- The user management functional module is to achieve the setting of user permission, verification of user's ID, modification of user's password, setting of evaluation time and update of relevant data.

- The data computing functional module is to calculate total points of the evaluation results, put results in order and generate comments.

- The search functional module enables students, teachers and employers to search the marks and comments of colleges according to different majors and departments, and search the rank list of various colleges.

- The index system management functional module is to achieve the entry, modification and deletion of relevant data in the evaluation index system.

- The printing functional module is to print the evaluation result and the evaluation index list.

\section{Database Design}

In accordance with the analysis and design of the function modules in the system, the relationship between user demand and all entities that can meet user demand can be figured out[4]. The major E-R relationship of teacher practice teaching evaluation is shown as figure 3.

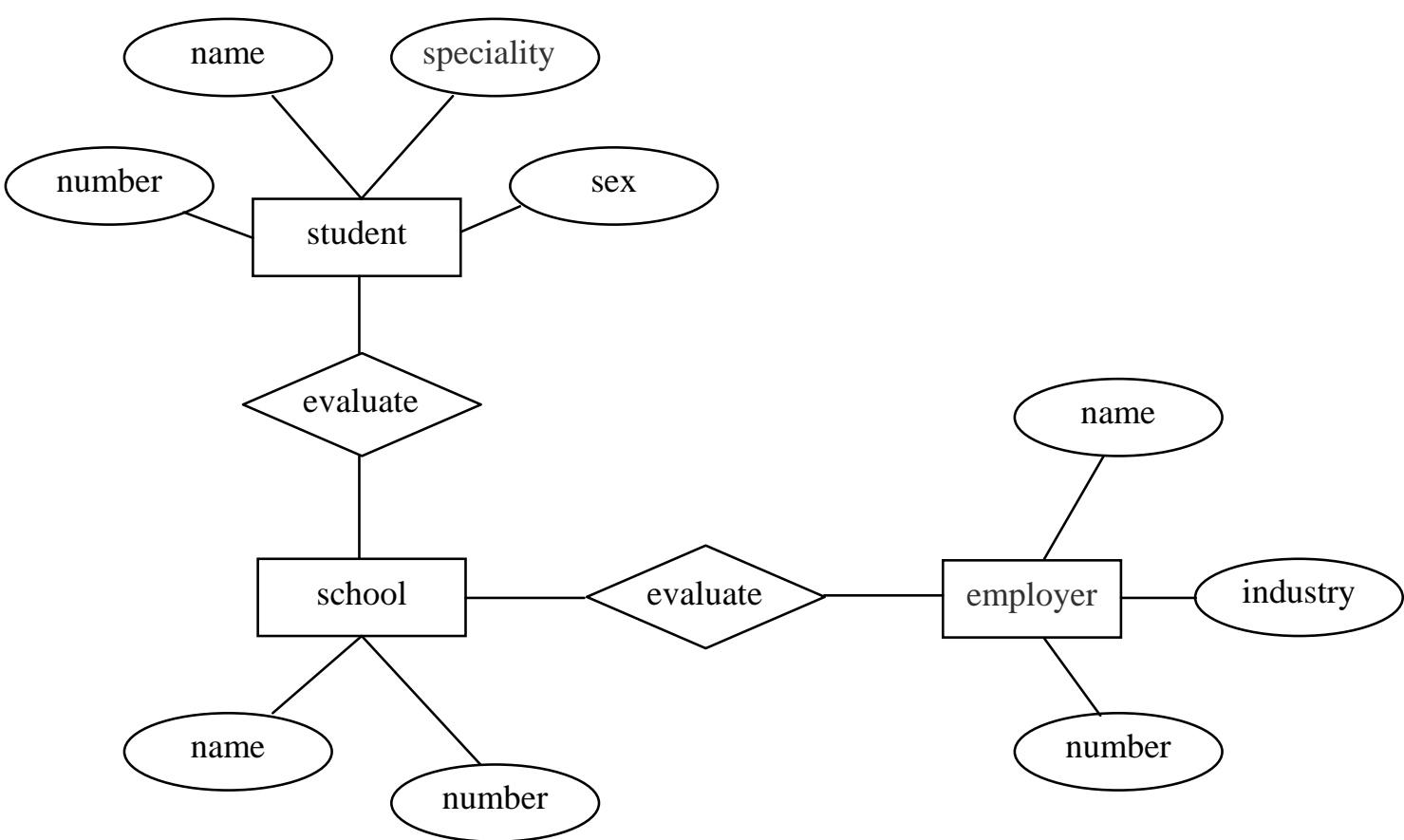

Figure 3. The major E-R relationship of internship evaluation

\section{Implementation of the system}

According to the above design strategy, ASP.NET and ADO.NET techniques are combined to employ the simple, stable, extensible and reusable programming model to generate the content in the Web browser. The work flow of the system is quite simple. Students log in the system to evaluate teachers' internship activities and give feedback. Teachers log in the system to self-evaluate and check students' feedback. Leaders log in the system to evaluate teachers' internship activities and check the evaluation result and students' feedback of all teachers.

The key technique to achieve a series of functions including evaluation of internship activities and information feedback is the database access technique. In the system, users get access to the database through ADO.NET technique[5]. DataSet is a very important core member. It is the cache of the data reflected in the local computer. Any operation of it is implemented within the computer cache. Even if the data link is cut off or the database is shut off, it is still useful. 
The code used to connect ADO.NET and DataSet is shown as follows:

SqlConnection sqlConnectionl $=$ new SqlConnection ("Data Source $=$ localhost ; Integrated Security = SSPI ; Initial Catalog = Northwind” );

// Create data connection

SqlCommand selectCMD = new SqlCommand ( " SELECT TeacherID ， TeacherName FROM Teachers” , sqlConnectionl );

// Create and initialize SqlCommand object

SqlDataAdapter sqlDataAdapter1 = new SqlDataAdapter ( ) ;

teachDA. SelectCommand = seletCMD ;

sqlConnection. Open ( ) ;

// Create SqlDataAdapter object, and according to SelectCommand attribute to retrieve data

DataSet dsDataSet1 $=$ new DataSet () ;

sqlDataAdapter1. Fill ( dsDataSet1 , “Teachers” ) ;

// Use SqlDataAdapter Fill DataSet

sqlConnection. Close ( ) ;

// Close the data connection

\section{Conclusions}

The evaluation system operation interface, easy to operate, stable operation, easy evaluators, including students, teachers, educational administrators to make a fair evaluation of each teacher's teaching; teaching evaluation results feedback, teachers can be comprehensive, in-depth understanding of own teaching, found problems exist in their own teaching, timely adjustment of teaching methods, use of practical measures to improve, improve the quality of teaching; teaching management departments at all levels is also conducive to a comprehensive, effective and systematic understanding of the school teacher's teaching work prominent teaching in the school's central position and role; improve the Educational Administrative teacher work efficiency, teaching evaluation network, digitization and automation.

\section{References}

[1] J. J. Chen, "Gao Yubin. Design and Realization of Instruction Evaluation Data Mining System Based on J2EE,”Computer Knowledge and Technology, vol. 14, pp.392-402, July 2011.

[2] C. Y. Wang,'Design of Teaching Quality Evaluation System Based on Structure of B /S," Journal of Lanzhou Petrochemical College of Vocational Technology, vol. 22, pp.65-67, April 2008.

[3] G. Teng. "Design and Realization of Teaching Evaluation System Based on.NET Platform," Electronic Engineer, vol. 12, pp.75-77, March 2008.

[4] Y. Wang, "Research and Construction of Practical Teaching Quality Management System in Colleges and Universities,"Experimental Technology and Management, vol. 15, pp.14-16, July 2012.

[5] L. P. Yin, “ Research on the Construction of Evaluation System of Practice Teaching in Higher Vocational Education,” Value Engineering, vol. 32, pp.265-267, Dec. 2013. 